\title{
On finite-time ruin probabilities with reinsurance cycles influenced by large claims
}

\author{
M. Bargès ${ }^{* \dagger}$, S. Loisel ${ }^{\dagger}$ and X. Venel ${ }^{\dagger \ddagger}$
}

May 14, 2011

\begin{abstract}
Market cycles play a great role in reinsurance. Cycle transitions are not independent from the claim arrival process : a large claim or a high number of claims may accelerate cycle transitions. To take this into account, a semi-Markovian risk model is proposed and analyzed. A refined Erlangization method is developed to compute the finite-time ruin probability of a reinsurance company. Numerical applications and comparisons to results obtained from simulation methods are given. The impact of dependency between claim amounts and phase changes is studied.
\end{abstract}

JEL classification codes: G22 C60

Keywords: Finite-time ruin probability, reinsurance cycles, Erlangization, dependence in risk theory, phase-type distributions.

\section{Introduction}

The classical Sparre-Anderson risk model describes the surplus process $\left\{R_{t}\right\}_{t \geqslant 0}$ for an insurance or a reinsurance company as $R_{0}=u$ and

$$
R_{t}=u+c t-S_{t},
$$

where $u$ represents the non-negative initial capital and $c>0$ is the premium income rate. The compound renewal process $\left\{S_{t}\right\}_{t \geqslant 0}$ represents the cumulated claim amount up to time $t$ and is

\footnotetext{
* Université de Lyon, Université Claude Bernard Lyon 1, Institut de Science Financière et d'Assurances, 50 Avenue Tony Garnier, F-69007 Lyon, France

†École d'Actuariat, Université Laval, Québec, Canada

$\ddagger$ Université Pierre et Marie Curie Paris 6, 4 place Jussieu 75005 Paris, France
} 
expressed as

$$
S_{t}=\left\{\begin{array}{l}
0, \text { if } N_{t}=0 \\
\sum_{i=1}^{N_{t}} X_{i} \text { otherwise }
\end{array}\right.
$$

where the claim amounts $X_{i}, i \geqslant 1$, are non-negative and i.i.d. random variables. The number of claims up to time $t$ is modeled by the renewal process $\left\{N_{t}\right\}_{t \geqslant 0}$ with i.i.d. inter-arrival times denoted by $W_{k}, k \geqslant 1$. The $X_{i}$ 's, $i \geqslant 1$ are independent from the process $\left(N_{t}\right)_{t \geqslant 0}$. One quantity of interest (among others) to set risk tolerance and risk limits is the finite-time ruin probability

$$
\psi(u, T)=P\left[\exists t \in[0, T], \quad u+c t-S_{t}<0\right], \quad u \geqslant 0, T \geqslant 0 .
$$

Different methods to compute this finite-time (non-)ruin have been proposed in the literature. Picard and Lefèvre (1997) derived an explicit formula for the finite-time non-ruin probability in a compound Poisson model where the claim amounts are integer-valued. This idea was then widely used and extended to more general models as in De Vylder and Goovaerts (1999), Ignatov and Kaishev (2004), Rullière and Loisel (2004) or Lefèvre and Loisel (2009), among others. Asymptotic methods have also been used to evaluate the finite-time ruin probability as in Biard et al. (2008) where the claim sizes are heavy-tailed and where stationarity and independence assumptions are relaxed in possible correlation crises. Previous general asymptotic results have been provided for sums of random variables as in Wüthrich (2003), Albrecher et al. (2006) or Kortschak and Albrecher (2009).

The method we focus on is called Erlangization. It was first introduced in Avram and Usabel (2003) but principally developed by Asmussen et al. (2002). The Erlangization method consists in replacing the deterministic time horizon $T$ by an Erlang-distributed random variable $\mathcal{H}$ with mean $T$ and independent from the claim process. Asmussen et al. (2002) have shown that the ruin probability before the independent Erlang time $\mathcal{H}$ coincides with the ruin probability in a certain Markovian fluid model with a matrix-exponential form. They considered the compound Poisson risk model with phase-type claim amounts. Stanford et al. (2005a) proposed an extension to the general Sparre-Anderson risk model. In Yu et al. (2007), the risk process with phase-type distributed interclaim times and claim amounts is perturbed by a standard Brownian motion. Ramaswami et al. (2008) also extended the Erlangization method to Markovian modulated fluid flow models which will prove to be essential our work. Erlangization has also been used in some other domains as in Stanford et al. (2005b) where the forestry problem of the evolution of uncontrolled fire perimeter over time is treated. For a complete review on fluid flow matrix analytic methods applied in ruin theory, see Badescu and Landriault (2009).

We propose here to consider the reinsurance market. In the reinsurance business, the presence of underwriting cycles cannot be ignored. As said in Cummins et al. (2008), reinsurance cycles are composed of soft phases, during which reinsurance supply is extensive and reinsurance premium income rates are quite low and decreasing, and hard phases where the supply is too low and the premium income rate becomes higher and higher. Phase transitions are hard to explain, and seem to happen due to both endogenous and exogenous events. In Weiss (2007), it is mentioned that these changes can come from institutional features as well as the effects of real phenomena. These phenomena can be asymmetric information in capital markets, capital surpluses and shortages in 
insurance or interest and/or loss shocks.

A simple generalization of the above-mentioned Erlangization model could be obtained with a Markovian environment process which would modulate the premium income rate, following the idea of Asmussen (1989) and of many others. Nevertheless, this first generalization leads to a model in which the cumulated claim amount process has no feedback control on the phases of the Markovian environment process $\left\{J_{t}\right\}$. This is not the case in real world, as changes in cycle phases may be triggered by a large claim. In this paper, we propose a generalization of the above risk model that takes into account this possible trigger.

We consider a simplification of a multi-phases cycle as it is exposed in Subsection 3.1 into a 2-phases reinsurance cycle consisting of a soft phase with low premium rate and a hard phase with high premium rate. In financial insurance, it is usual to suppose only two environment states as in the model introduced by Hardy (2001) where the volatility of a stock is either high or low according to the state environment. Considering two environment phases permits of course not only model simplifications but also avoids many estimation problems which appear when more phases are assumed.

In the Solvency II framework, the capital required to cover risk is determined in such a way that a 1-year Value-at-Risk is controlled. Nevertheless, for reinsurance risk, and in particular for catastrophe risk, 1-year occurrence probabilities of extreme events are small, and risk must be controlled within a larger time period, 5 to 10 years, to determine not only solvency requirements but also long term economic capital in internal risk models. The average length of reinsurance cycles typically ranges from 4 to 10 years, see Venezian (1985), Cummins and Outreville (1987) and Weiss (2007). It is thus necessary to use a risk model that incorporates this cyclic behavior.

Even if the numerical application provided in Section 6 is detailed when only the premium income rates changes according to the cycle phases, our model is developed for the inter-claim time and claim amount distributions to be dependent from the environment cycle process. The feedback of the claim amounts on the environment process thus involves some dependence between inter-claim times and claim amounts. Other types of dependence have already been explored in the study of infinite- or finite-time ruin probability. Boudreault et al. (2006) and Albrecher and Boxma (2004) assumed that each claim amount depends on the previous inter-claim time or inversely that each inter-claim time depend on the previous claim amount. This has been extended to claim amounts depending on several preceding inter-claim times as can be seen in Biard et al. (2011). In Albrecher and Teugels (2006) the dependence between the inter-claim times and claim amounts is introduced by an arbitrary copula.

One of the contributions of the paper is to propose a more suitable model for long term economic capital management for reinsurance companies. Within this framework, the dependence of the premium income rate, the claim amount distribution and the inter-claim time distribution with the cycle environment is taken into account. The model also permits a feedback control from the claim amounts on the cycle phases. We adapt the Erlangization method to this context in order to approximate the finite-time ruin probability. We use intensive calculation techniques to this aim and compare our results to Monte-Carlo simulations. We evaluate the model error in several examples and quantify the impact of the claim amounts feedback over a 5-years time horizon. We 
show that for some reasonable values, the finite-time ruin probability observes a decrease of $6 \%$ when this correlation is not considered. This is of course just an illustrative example, and not a general fact.

This paper begins with a recall on the Erlangization method and related results. In Section 3 reinsurance cycles are discussed. General notations are given and a first naive model is proposed where we show how the Erlangization method can be adapted to deterministic phase changes. The main model incorporating the impact of the claim amounts on the change in cycle phases is presented in Section 4. Section 5 is dedicated to the control of the precisions of the proposed main model. Finally, an extended and practical illustration is provided in Section 6 where fitting methods and finite-time ruin probability approximations are given.

\section{Erlangization method}

We recall in this section the principle of the Erlangization method and the central theorems from Asmussen et al. (2002) which lead to an approximation for the finite-time ruin probability. We follow the presentation of Asmussen et al. (2002) and Ramaswami et al. (2008) and slightly adapt the notation for it to be consistent with our purpose. First, the surplus process $R_{t}$ is embedded in an initial continuous Markovian additive fluid process $\left\{\left(\tilde{J}_{t}, \tilde{V}_{t}\right)\right\}$ where $\tilde{J}_{t}$ is a fluid flow and $\tilde{V}_{t}$ a Markovian environment process. The state space $\tilde{E}$ of $\tilde{V}_{t}$ consists of two subspaces $\tilde{E}_{-}$and $\tilde{E}_{+}$ such that when $\tilde{J}_{t}$ is in state $i \in \tilde{E}_{-}, \tilde{V}_{t}$ is in a decreasing inter-claim time state (no jump) with fluid rate $r_{i}=-c_{i}$ and when $\tilde{J}_{t}$ is in state $i \in \tilde{E}_{+}, \tilde{V}_{t}$ is in an increasing claim state (jump) with fluid rate $r_{i}=1$. The transition intensity matrix of $\tilde{J}_{t}$ is noted as

$$
\Lambda=\left(\begin{array}{ll}
\Lambda_{--} & \Lambda_{-+} \\
\Lambda_{+-} & \Lambda_{++}
\end{array}\right)
$$

where $\Lambda_{i_{1} i_{2}}$ correspond to the transition from a state of subspace $\tilde{E}_{i_{1}}$ to a state of subspace $\tilde{E}_{i_{2}}$ with $i_{1}$ and $i_{2} \in\{-,+\}$.

The aim of the Erlangization method is to evaluate the finite-time ruin probability before an Erlang horizon $\mathcal{H} \sim \operatorname{Erlang}(a, L)$. The finite-time ruin probability can thus be seen as the probability that the fluid flow reaches the initial reserve $u$ in an increasing state (claim state) conditioned on starting in a decreasing state (inter-claim time state) before the expiry of the Erlang horizon $\mathcal{H}$. For this purpose, we construct an expanded absorbing Markovian fluid process $\left\{\left(J_{t}, V_{t}\right)\right\}$. The state space $E$ of the Markovian environment process $J_{t}$ is now composed of three subsets $E_{0}, E_{-}$and $E_{+}$where $E_{0}$ is the one state absorbing space, $E_{-}$is the decreasing inter-claim time state space and $E_{+}$is the increasing claim state space.

The transition intensity matrix for the Markovian environment process $J_{t}$ is

$$
\widehat{\Theta}=\left(\begin{array}{ccc}
0 & 0 & 0 \\
\widehat{h}_{-} & H \oplus \Lambda_{--} & I_{L} \otimes \Lambda_{-+} \\
0 & I_{L} \otimes \Lambda_{+-} & I_{L} \otimes \Lambda_{++}
\end{array}\right)=\left(\begin{array}{ccc}
0 & 0 & 0 \\
\widehat{h}_{-} & \widehat{\Theta}_{--} & \widehat{\Theta}_{-+} \\
0 & \widehat{\Theta}_{+-} & \widehat{\Theta}_{++}
\end{array}\right),
$$


where $H$ is the phase generator of the Erlang horizon $\mathcal{H}$ and $\widehat{h}_{-}$is the vector of absorbtion probabilities for the fluid process in a decreasing state. Operators $\otimes$ and $\oplus$ respectively denote the Kronecker product and the Kronecker sum.

The incorporation of the premium income rates which correspond to the fluid rates for the fluid process $V_{t}$ is done by time scaling. These fluid rates are $r_{i}=0$ for $i \in E_{0}, r_{i}=-c_{i}$ for $i \in E_{-}$and $r_{i}=1$ for $i \in E_{+}$. The matrix $\Theta$ which takes the premium income rates into account associated with $\widehat{\Theta}$ is

$$
\begin{aligned}
\Theta & =\left(\begin{array}{ccc}
0 & 0 & 0 \\
\left(I_{L} \otimes R_{-}\right)^{-1} \times \widehat{h}_{-} & \left(I_{L} \otimes R_{-}\right)^{-1} \times \widehat{\Theta}_{--} & \left(I_{L} \otimes R_{-}\right)^{-1} \times \widehat{\Theta}_{-+} \\
0 & \widehat{\Theta}_{+-} & \widehat{\Theta}_{++}
\end{array}\right) \\
& =\left(\begin{array}{ccc}
0 & 0 & 0 \\
\left(C_{-}\right)^{-1} \times \widehat{h}_{-} & \left(C_{-}\right)^{-1} \times \widehat{\Theta}_{--} & \left(C_{-}\right)^{-1} \times \widehat{\Theta}_{-+} \\
0 & \widehat{\Theta}_{+-} & \widehat{\Theta}_{++}
\end{array}\right) \\
& =\left(\begin{array}{ccc}
0 & 0 & 0 \\
h_{-} & \Theta_{--} & \Theta_{-+} \\
0 & \Theta_{+-} & \Theta_{++}
\end{array}\right),
\end{aligned}
$$

where $R_{-}$is a diagonal matrix formed with the decreasing fluid rates.

Denote by $\eta_{i j}$ the probability that when $J_{0}=i \in E_{-}$, then the first upcrossing of level 0 of $\left\{V_{t}\right\}$ occurs at time $t$ with $J_{t}=j \in E_{+}$. The matrix $\eta$ corresponds to the matrix of the "upcrossing phase probabilities" at the completion of a downwards excursion for $\left\{V_{t}\right\}$, conditioned on starting in a decreasing state. We now cite some results from Asmussen et al. (2002), which can also be seen in Ramaswami et al. (2008) which aim to give an evaluation for the probability $\psi(u, \mathcal{H})$ of reaching $u$ conditioned on starting in a decreasing state before the expiry of the Erlang horizon. We first reccal a general result on the upcrossing probabilities of fluid models.

Theorem 1 Let $\left\{\left(J_{t}, V_{t}\right)\right\}$ be a Markovian fluid process and $\Theta$ the transition intensity matrix of the Markovian environment process $\left\{J_{t}\right\}$. Let also $\eta$ denote the "upcrossing phase probabilities" at the completion of a downwards excursion for $\left\{V_{t}\right\}$, conditioned on starting in a decreasing state $i \in E_{-}$. Note by $\psi(u)$ the vector of the infinite horizon phase-distribution at $u$ (the initial surplus, starting at 0), conditioned on starting in a decreasing state. Then we have :

(a) the matrix $\eta$ satisfies the Riccati equation:

$$
\eta \Theta^{++}+\eta \Theta^{+-} \eta+\Theta^{--} \eta+\Theta^{-+}=0
$$

and

$$
\psi(u)=\eta e^{U u} 1
$$

where

$$
U=\Theta^{++}+\Theta^{+-} \eta
$$

(b) let now $\delta>0$ be a "convergence improving parameter", satisfying $\delta>\max \left(-\left(\Theta^{--}\right)_{i i}\right)$ for all state $i$ of $\mathcal{H}$. Then, the solution $\eta$ of the previous Riccati equation can be computed by the iteration 
scheme $\eta=\lim _{n \rightarrow \infty} \eta^{(n)}$ where $\eta^{(0)}=0$,

$$
\eta^{(n+1)}=\left(\left(\delta I+\Theta^{--}\right) \eta^{(n)}+\Theta^{-+}\right)\left(\delta I-\left(\Theta^{++}+\Theta^{+-} \eta^{(n)}\right)\right)^{-1} .
$$

Focusing on our case where we look at the fluid process before the expiry of the Erlang horizon time $\mathcal{H}$ with initial probability vector $\gamma$, we have the following corollary.

Corollary 2 Including the Erlang time $\mathcal{H}$ with the initial probability vector $\gamma$, it follows that

$$
\psi(u, \mathcal{H})=\gamma \eta e^{V u} 1
$$

where $V=\Theta^{++}+\Theta^{+-} \eta$.

Finally, Asmussen et al. (2002) obtained the following convergence result.

Theorem 3 For $T>0, \mathcal{H}$ the Erlang distribution with $L$ stages and mean $T$, then

$$
\psi(u, \mathcal{H}) \rightarrow \psi(u, T) \text { as } L \rightarrow \infty \text {, at rate } O\left(L^{-1}\right) .
$$

The proof of Theorem 1 is based on the fluid flow theory and on the study of the steadystate distribution of the general fluid process $\left\{\left(V_{t}, J_{t}\right)\right\}$. Indeed, Asmussen (1995) proved that the distribution of the steady-state variable $(V, J)$ is described by $V$ being phase-type given $J=i$, with phase generator $U$ independent from $i$, the unit column vector $e$ as initial vector for $i \in E_{+}$and $\eta_{i}$ as the $i$ th row of $\eta$, for $i \in E_{-}$. The Corollary 2 is then deduced when including the Erlang time horizon. The details of the proof of Theorem 3 can be found in Asmussen et al. (2002).

We show in appendix how to deal with the matrix inversions that are involved in Equation (3).

\section{A few words on reinsurance cycles modeling}

\subsection{Reinsurance cycles}

In order to give an idea of a possible generalization of the model we propose in this paper, we first expose here a cycle modeling with several phases. Then we recall the reasons of our simplification choice to a 2-phases cycle.

Reinsurance cycles can be composed of several phases with soft phases where the premium rates

are low and hard phases with high premium rates. Intermediate phases have premium rates varying according to their proximity with the soft or the hard phases. We suppose that a reinsurance cycle has, for example, 10 phases where $i=1$ corresponds to the softest phase and $i=10$ to the hardest 
phase. We note $c_{i}, i=1, \ldots, 10$, the premium income rate for the 10 phases with $c_{1} \leqslant c_{2} \leqslant \ldots \leqslant c_{10}$ and assume that when the cycle is in phase $i$, it could only go to phase $i-1, i+1, i+2$ or stay at $i$ depending on the claim amount. When a large claim occurs, the cycle have high probability to go to phase $i+1$ and if the claim is very large, the cycle can also directly jump to phase $i+2$. Figure 1 shows a possible cycle phase path using these ideas.

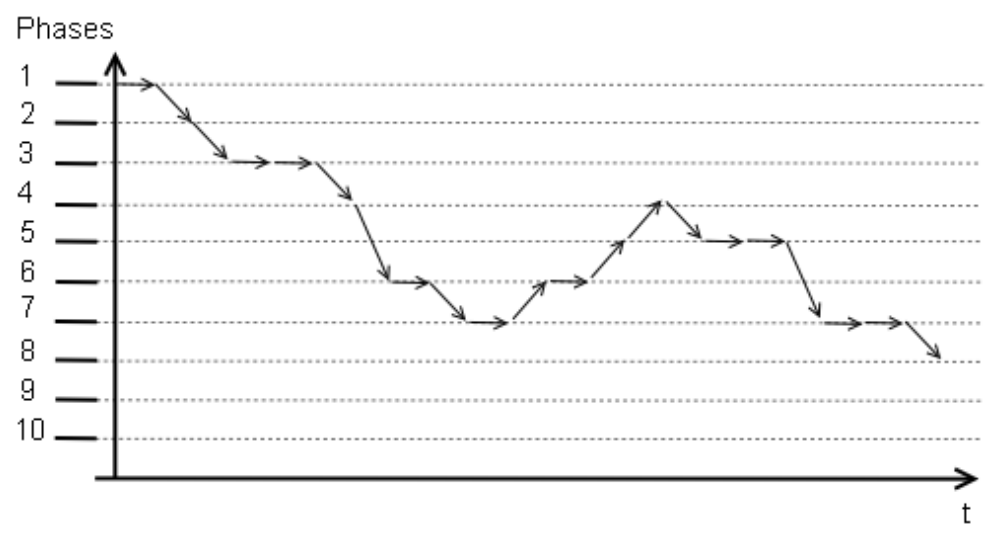

Figure 1: Example of a cycle path with 10 phases

We could also double the phase states such as $1^{\downarrow}, 1^{\uparrow}, 2^{\downarrow}, 2^{\uparrow}, 3^{\downarrow}, 3^{\uparrow}, \ldots$ (or $1,2^{\downarrow}, 2^{\uparrow}, 3^{\downarrow}, 3^{\uparrow}, \ldots$, 10 ) in order to keep the general trend of the cycle. For example, we could set probabilities at state 3 according to the cycle trend as in Table 1.

\begin{tabular}{|r|c||r|c|}
\hline Phase $3 \downarrow$ to & Probability & Phase $3^{\uparrow}$ to & Probability \\
\hline Phase 2 & 0.001 & Phase 2 & 0.09 \\
Phase 3 & 0.9 & Phase 3 & 0.9 \\
Phase 4 & 0.09 & Phase 4 & 0.009 \\
Phase 5 & 0.009 & Phase 5 & 0.001 \\
\hline
\end{tabular}

Table 1: Example of phase change probabilities at phase 3.

But, in this paper, we focus our study on a reinsurance cycle that is composed of only a soft phase with low premium rate and a hard phase with high premium rate. Indeed, dealing with models with more than two phases in a cycle would require very large transition matrices and would highly increase computational issues. Moreover, it is conventional in finance insurance to consider environment processes with 2 states, as in Hardy (2001), which furthermore avoids many estimation problems.

We assume that large claims have high probabilities to cause a change from the soft phase to the hard phase. This impact of the claim amounts on the environment process represents quite well the general reinsurance loss market as most of insurance and reinsurance companies hold high expositions in the most risky regions (Western Europe, Japan, Eastern United-States, South-East Asia, China,...). In consequence, a big event (catastrophe,...) impacts all the actors with a very large claim. 


\subsection{General notation}

We fix now some notation for all the following sections. We suppose that in each cycle phase the claim amounts are i.i.d. and phase-type distributed such as $X_{i} \sim P H\left(G_{i}, \alpha_{i}\right)$ where $G_{i}$ is the phase generator matrix and $\alpha_{i}$ the initial row vector in cycle phase $i$. The inter-claim times are also i.i.d. and phase-type distributed. The inter-claim time random variable for cycle phase $i$ is noted as $W_{i} \sim P H\left(K_{i}, \beta_{i}\right)$ with $K_{i}$ the phase generator matrix and $\beta_{i}$ the initial row vector. We write by $g_{i}=-G_{i} e$ and $k_{i}=-K_{i} e$ the rates of absorption for respectively $X_{i}$ and $W_{i}$ where $e$ is the unit column vector. Denote by $T$ the fixed time horizon. The phase-type parametrization of the Erlang horizon time $\mathcal{H}$ with $L$ stages, mean $T$ and rate $a=L / T$ is $\mathcal{H} \sim P H(H, \gamma=(1,0, \ldots, 0))$ where

$$
H=\left(\begin{array}{cccc}
-a & a & 0 & 0 \\
0 & \ddots & \ddots & 0 \\
0 & 0 & -a & a \\
0 & 0 & 0 & -a
\end{array}\right)
$$

\subsection{Deterministic phase changes}

We give first a naive model which assumes that only one change of phase happens and that this time of phase change is known in advance. We present here the case where the cycle environment process begins in the soft phase and switches to the hard phase at a deterministic time $T_{1}$ and stops at the horizon $T$. But this model could be adapted with the cycle beginning in the hard phase and going to the soft phase. The times spent in the soft phase and in the hard phase are respectively $T_{1}$ and $T_{2}=T-T_{1}$. Here, the Erlang horizon time $\mathcal{H}$ with mean $T$ and $L$ stages is the sum of two intermediate Erlang horizon times $\mathcal{H}_{1}$ and $\mathcal{H}_{2}$ with means respectively $T_{1}$ and $T_{2}$ and $L_{1}$ and $L_{2}$ stages. We can then write the transition intensity matrix $\widehat{\Theta}$ as

$$
\begin{aligned}
\widehat{\Theta} & =\left(\begin{array}{ccc}
0 & 0 & 0 \\
\widehat{h}_{-} & \widehat{\Theta}_{--} & \widehat{\Theta}_{-+} \\
0 & \widehat{\Theta}_{+-} & \widehat{\Theta}_{++}
\end{array}\right) \\
& =\left(\begin{array}{ccccc}
0 & 0 & 0 & 0 & 0 \\
0 & H_{1} \oplus K_{1} & M_{12} & I d_{L_{1}} \otimes k_{1} \alpha_{1} & 0 \\
h_{2} \otimes e_{k_{2}} & 0 & H_{2} \oplus K_{2} & 0 & I d_{L_{2}} \otimes k_{2} \alpha_{2} \\
0 & I d_{L_{1}} \otimes g_{1} \beta_{1} & 0 & I d_{L_{1}} \otimes G_{1} & 0 \\
0 & 0 & I d_{L_{2}} \otimes g_{2} \beta_{2} & 0 & I d_{L_{2}} \otimes G_{2}
\end{array}\right),
\end{aligned}
$$

where $M_{12}=\left(\left(h_{1} \times \gamma_{2}\right) \times e_{k_{1}}\right) \times \beta_{2}$ is the bloc matrix of transitions between the two phases and $h_{i}=-H_{i} e$ the absorption rate vector for the phase-type parametrization of the Erlang horizon $\mathcal{H}_{i}$.

Remark 4 The way to determine the numbers of stages $L_{1}$ and $L_{2}$ for respectively $\mathcal{H}_{1}$ and $\mathcal{H}_{2}$ is shown in appendix. 
Example 5 Consider that $H_{1} \sim \operatorname{Erlang}(1, a)$ and $H_{2} \sim \operatorname{Erlang}(2, a)$, then we have

$$
\widehat{\Theta}=\left(\begin{array}{ccccccc}
0 & 0 & 0 & 0 & 0 & 0 & 0 \\
0 & K_{1}-a I d_{n_{1}} & \left(a \times e_{n_{1}}\right) \times \beta_{2} & 0 & k_{1} \alpha_{1} & 0 & 0 \\
0 & 0 & K_{2}-a I d_{n_{2}} & a I d_{n_{2}} & 0 & k_{2} \alpha_{2} & 0 \\
a e_{n_{2}} & 0 & 0 & K_{2}-a I d_{n_{2}} & 0 & 0 & k_{2} \alpha_{2} \\
0 & g_{1} \beta_{1} & 0 & 0 & G_{1} & 0 & 0 \\
0 & 0 & g_{2} \beta_{2} & 0 & 0 & G_{2} & 0 \\
0 & 0 & 0 & g_{2} \beta_{2} & 0 & 0 & G_{2}
\end{array}\right)
$$

where $n_{1}$ is the number of stages of $W_{1}$ and $n_{2}$ the number of stages of $W_{2}$.

Using the same idea, it is also possible to construct an intensity matrix $\widehat{\Theta}$ for the situation where the environment process begins in the hard phase and goes to the soft phase at a deterministic time. But in fact, cycle phase changes are random and may be influenced by large claims.

\section{Phase change instants dependent from claim amounts}

We now focus on the main model of the article where we let the cycle switch from the soft phase to the hard phase when a claim amount is greater than a threshold $x_{0} \geqslant 0$. In what follows, we suppose that the cycle begins in the soft phase but using the idea of the previous simple model, one can add an initial hard phase with a deterministic length. As noted in introduction, the going back to the soft phase should also depend from the claim amount, for example according to the severity of the claim that caused the passing to the hard phase. But for simplification reasons, we suppose here that it happens after a deterministic time $t_{0} \geqslant 0$. The fact that the time spent in the previous soft phase is not known, we can not proceed as in the deterministic phase change model developed above.

We require here that the claim amounts are distributed as mixtures of Erlang distributions. Mixtures of Erlang distributions include the exponential distribution, the Erlang distribution and mixtures of Exponential distributions. The canonical random variable for the claim amounts in cycle phase $i, i=1,2$, is then written as

$$
X_{i}=\sum_{j=1}^{n_{i}} \alpha_{i j} Y_{i j},
$$

where $Y_{i j}$ are $n_{i}$ independent and Erlang distributed random variables such that $Y_{i j} \sim \operatorname{Erlang}\left(m_{i j}, \lambda_{i j}\right)$ with shape parameters $m_{i j}>0$ and rate parameters $\lambda_{i j}>0$. The scalars $\alpha_{i j} \geqslant 0$ are the mixture proportions and must satisfy $\alpha_{i 1}+\ldots+\alpha_{i n_{i}}=1$. We assume that $E\left[Y_{i 1}\right]=m_{i 1} / \lambda_{i 1}>\ldots>E\left[Y_{i n_{i}}\right]=$ $m_{i n_{i}} / \lambda_{i n_{i}}$.

This section is decomposed into 2 subsections. The first one introduces the transition from the soft phase to the hard phase which depends on the claim amounts. The second subsection exposes the way the go back to the soft phase. 


\subsection{From the soft phase to the hard phase}

We first introduce the transition from the soft phase to the hard phase which is dependent from the claim amounts. To modelize the fact that the environment process goes to the hard phase when a claim amount is greater than the threshold $x_{0}$, we use the probabilities

$$
p_{1 j}=P\left(Y_{1 j}>x_{0}\right)=1-F_{Y_{1 j}}\left(x_{0}\right)
$$

where $Y_{1 j} \sim \operatorname{Erlang}\left(m_{1 j}, \lambda_{1 j}\right)$, for $j=1, \ldots, n_{1}$. As the random variables $Y_{1 j}$ are ordered by decreasing mean, we have $p_{11}>\ldots>p_{1 n_{1}}$. Then we integrate these probabilities into the transition matrix $\Lambda$ of the initial Markov process $\left\{I_{t}\right\}$ as follows. Construct line vectors $\widehat{p}_{1 j}=\left(0, \ldots, 0, p_{1 j}\right)$ of length $m_{1 j}$ and concatenate them in $p_{1}=\left(\widehat{p}_{11}, \ldots, \widehat{p}_{1 n_{1}}\right)$. Then, set

$$
P_{1}=\left(p_{1}^{\prime} \otimes e_{k_{1}}\right),
$$

the matrix containing the probabilities for the claim amounts to be greater than $x_{0}$ when the environment process is in the soft phase as needed to incorporate them into $\Lambda$. The latter becomes

$$
\Lambda=\left(\begin{array}{cccc}
K_{1} & 0 & k_{1} \alpha_{1} & 0 \\
0 & K_{2} & 0 & k_{2} \alpha_{2} \\
P_{1}^{-} \odot g_{1} \beta_{1} & P_{1} \odot g_{1} \beta_{2} & G_{1} & 0 \\
0 & g_{2} \beta_{2} & 0 & G_{2}
\end{array}\right)
$$

where

$$
P_{1}^{-}=\left(\left(e_{n_{1}}-p_{1}\right)^{\prime} \otimes e_{k_{1}}\right),
$$

and the operator $\odot$ is the Hadamard product (entrywise product). Thus the transition intensity matrix $\widehat{\Theta}$ of $\left\{J_{t}\right\}$ can be obtained as exposed in Section 2. The concept of dependent changes is here probabilistically respected to approximate the ruin probability.

\subsection{From the hard phase to the soft phase}

We now include the fact that the cycle process can go back to the soft phase from the hard phase. As said previously, we do not suppose that this transition is dependent from the claim amount but that the expected time spent in the hard phase is fixed at time $t_{0}$. This transition can happen at any state of the Erlang time $\mathcal{H}$ when the process is in the hard phase. The possibility to go from the hard phase to the soft phase is introduced by a transition probability $q$ in the block $\widehat{\Theta}_{--}$of the Markovian process $\left\{J_{t}\right\}$. It corresponds to the probability of changing from the hard phase to the soft phase at any state of the Erlang time $\mathcal{H}$ and is given as follows.

Note by $Z$ the number of Bernoulli trials with success probability $q$ needed to get one change from the hard phase to the soft phase. The random variable $Z$ is indeed geometrically distributed

with mean $\frac{1}{q}$. We set the expected value of $Z$ equal to the number of stages of the Erlang time $\mathcal{H}$ 
at which we want the cycle to jump to the soft phase. We write this as

$$
E[Z]=a \times t_{0}
$$

which ensures that the time spent in the hard phase is $t_{0}$ on average. The rate parameter $a$ of the Erlang time $\mathcal{H}$ corresponds to the number of states needed to have a one year long time. We thus deduce the probability $q$ as

$$
q=\frac{1}{a \times t_{0}}=\frac{T}{L \times t_{0}} .
$$

For a better overview, suppose that $\mathcal{H} \sim \operatorname{Erlang}(L, a)$ with $L=2$ and that the inter-claim times $W_{1}$ and $W_{2}$ are identically distributed. Their canonical random variable $W$ is distributed as a mixture of exponential distributions with intensity matrix $K$ such as

$$
K=\left(\begin{array}{cc}
-\lambda_{1} & 0 \\
0 & -\lambda_{2}
\end{array}\right)
$$

The block $\widehat{\Theta}_{--}$becomes

$$
\begin{aligned}
\widehat{\Theta}_{--}= & \left(\begin{array}{cccc|cccc}
-\lambda_{1}-a & 0 & 0 & 0 & a & 0 & 0 & 0 \\
0 & -\lambda_{2}-a & 0 & 0 & 0 & a & 0 & 0 \\
0 & 0 & -\lambda_{1}-a & 0 & a q & 0 & a(1-q) & 0 \\
0 & 0 & 0 & -\lambda_{2}-a & 0 & a q & 0 & a(1-q) \\
\hline 0 & 0 & 0 & 0 & -\lambda_{1}-a & 0 & 0 & 0 \\
0 & 0 & 0 & 0 & 0 & -\lambda_{2}-a & 0 & 0 \\
0 & 0 & 0 & 0 & 0 & 0 & -\lambda_{1}-a & 0 \\
0 & 0 & 0 & 0 & 0 & 0 & 0 & -\lambda_{2}-a
\end{array}\right) \\
& =\left(\begin{array}{cc}
\Lambda_{--}-a I & M^{*} \\
0 & \Lambda_{--}-a I
\end{array}\right) .
\end{aligned}
$$

The environment process can go from the hard phase to the soft phase without affecting the run of the Erlang time $\mathcal{H}$. This can of course be extended for $L \geqslant 2$ leading to a block matrix

$$
\widehat{\Theta}_{--}=\left(\begin{array}{ccccccc}
\Lambda_{--}-a I & M^{*} & 0 & 0 & \ldots & 0 & 0 \\
0 & \Lambda_{--}-a I & M^{*} & 0 & \ldots & 0 & 0 \\
\cdots & \ldots & \cdots & \ldots & \cdots & \ldots & \ldots \\
0 & 0 & 0 & 0 & \cdots & \Lambda_{--}-a I & M^{*} \\
0 & 0 & 0 & 0 & \cdots & 0 & \Lambda_{--}-a I
\end{array}\right)
$$

where the block $M^{*}$ contains the transition probabilities from the hard phase to the soft phase.

\subsection{Computational aspects}

Ramaswami et al. (2008) proposed a recursion procedure to solve Equation (2) in $\eta$ using its particular structure which permits to reduce the dimension of the matrices involved in Theorem 1 
and gain significative computational time. Indeed, the upcrossing phase probability matrix $\eta$ has the following upper triangular block Toeplitz structure

$$
\eta=\left(\begin{array}{ccccc}
\eta_{0} & \eta_{1} & \eta_{2} & \cdots & \eta_{L-1} \\
0 & \eta_{0} & \eta_{1} & \cdots & \eta_{L-2} \\
0 & 0 & \eta_{0} & \cdots & \eta_{L-3} \\
\cdots & \cdots & \cdots & \cdots & \cdots \\
0 & 0 & 0 & \cdots & \eta_{0}
\end{array}\right)
$$

Denote that in our model, the block $\widehat{\Theta}_{--}$of the intensity matrix $\Theta$ can be expressed as

$$
\widehat{\Theta}_{--}=\left(\begin{array}{ccccccc}
\Lambda_{--}-a I & M^{*} & 0 & 0 & \cdots & 0 & 0 \\
0 & \Lambda_{--}-a I & M^{*} & 0 & \cdots & 0 & 0 \\
\ldots & \ldots & \cdots & \cdots & \cdots & \ldots & \ldots \\
0 & 0 & 0 & 0 & \cdots & \Lambda_{--}-a I & M^{*} \\
0 & 0 & 0 & 0 & \cdots & 0 & \Lambda_{--}-a I
\end{array}\right)
$$

where the block $M^{*}$ contains the transition probabilities between the two phases as explained above. Following the idea of Ramaswami et al. (2008), writing out Equation (2) leads to the recursion equations for the blocks of $\eta$

$$
\begin{aligned}
& \eta_{0} \Lambda_{+-} \eta_{0}+R_{--}^{-1}\left(\Lambda_{--}-a I\right) \eta_{0}+\eta_{0} \Lambda_{++}+R_{--}^{-1} \Lambda_{-+}=0 \\
& \eta_{l} \Lambda_{++}+\sum_{j=0}^{l} \eta_{j} \Lambda_{+-} \eta_{l-j}+R_{--}^{-1}\left(\Lambda_{--}-a I\right) \eta_{l}+R_{--}^{-1} M^{*} \eta_{l-1}=0 ; \quad l=1, \ldots, L-1 .
\end{aligned}
$$

Define for a matrix $A$ the vector $\operatorname{vec}(A)$ as the vector obtained by concatenating successive columns of $A$ from a single matrix. From Graham (1981), we can write for a matrix product $A X B$ that

$$
\operatorname{vec}(A X B)=\left(B^{t} \otimes A\right) \operatorname{vec}(X),
$$

where $B^{t}$ is the transpose of the matrix $B$. As in Ramaswami et al. (2008), we use this notation and Equations (7) to derive a recursive expression for $\eta_{l}, l=1, \ldots, L-1$ and obtain

$$
\begin{aligned}
\operatorname{vec}\left(\eta_{l}\right)= & \left\{\left[\Lambda_{++}+\Lambda_{+-} \eta_{0}\right]^{t} \otimes I+I \otimes\left[R_{--}^{-1}\left(\Lambda_{--}-a I\right)+\eta_{0} \Lambda_{+-}\right]\right\}^{-1} \\
& \times \operatorname{vec}\left(-\left[R_{--}^{-1} M^{*} \eta_{l-1}+\sum_{j=1}^{l-1} \eta_{j} \Lambda_{+-} \eta_{l-j}\right]\right) .
\end{aligned}
$$

Then, we only need to solve the nonlinear equation (6) using Theorem 1 to approximate $\eta_{0}$ and use the recursive expression (8) for $\eta_{l}, l=1, \ldots, L-1$ in order to derive an approximation of $\eta$. 


\section{Control of precisions}

The control of precisions of our approximation method is based on the following points : the number $L$ of stages of the Erlang horizon $\mathcal{H}$, the cycle change probabilities and the fitting algorithms that we use to obtain claim amounts distributed as mixtures of Erlang distributions..

\subsection{Model errors}

As shown is Theorem 6 of Asmussen et al. (2002), the following convergence result for the finite-time ruin probability stands:

$$
\psi\left(u, H_{L}\right)=\psi(u, T)+\frac{\psi^{(2)}(u, T)}{L 2 !}+O\left(L^{-2}\right),
$$

where $T>0$ and $H_{L}$ denote the Erlang distribution for $\mathcal{H}$ with $L$ stages and mean $T$. The model error can be estimated using the numerical computation for the second derivative of $\psi(u, T)$ as

$$
\psi^{(2)}(u, T)=\frac{\psi(u, T+h)+2 \psi(u, T)-\psi(u, T-h)}{h^{2}}+O\left(h^{2}\right)
$$

The same authors also suggest to use the Richardson extrapolation leading to the improved estimate

$$
\psi(u, T) \approx(L+1) \psi\left(u, H_{L+1}\right)-L \psi\left(u, H_{L}\right)
$$

with an refined convergence rate $L^{-2}$.

Using probabilities $p_{1 j}=\operatorname{Pr}\left(Y_{1 j}>x_{0}\right), j=1, \ldots, n_{1}$, as probabilistic modeling for the cycle environment to change from the soft phase to the hard phase can provide some errors. Indeed, the closer to 0 or to 1 all the $p_{1 j}$ 's, the most efficient the approximation. So, the threshold $x_{0}$ must be appropriately fixed. Giving such phase change probabilities leads to consider the $Y_{1 j}$ as if they are separate random variables. Medium values for the $p_{1 j}$ 's includes some perturbations and the modeling of the general claim behavior can fail.

The change of the environment process from the hard phase to the soft phase as explained in the Subsection 4.2 introduces a possible change of phase even when the process enters in the hard phase in the $t_{0}$ last years of the horizon time $T$. There, the process should not be able to change the phase but setting different probabilities for the phase change breaks the repeating block structure of $\eta$ and then the recursion equation (8) could not be used anymore. We suggest then to use the possibility for the environment process to go back to the soft phase only for a large horizon time $T$ and a large ratio $T / t_{0}$.

An other part of the potential error comes from the fitting algorithm. To ensure a good fit, several statistical tools such as Chi-squared test, Kolmogorov-Smirnov test, qq- and pp-plots can 
be used.

\section{$5.2 \quad$ Numerical errors}

Some numerical issues can also include some deviation as the matrix inversion required in Equation (3). This matrix inversion is done using the matrix right division operator "/" in Matlab R2007a software. Furthermore, we use the software package Expokit from R. B. Sidje (see Sidje (1998)) which uses Krylov subspace projection methods in order two compute the matrix exponential of Equation (4). The resort to this numerical tool can also include a slight deviation but permits to save substantial computation time.

\section{$6 \quad$ Numerical applications}

We illustrate in this section the use of our model when it is considered that the phase change instants depend on the claim amounts. As discussed in introduction, the European Solvency II regulation project will impose the insurance and reinsurance companies to have a 1-year horizon time ruin probability less than a certain threshold which is fixed at $0.5 \%$. For reinsurance companies dealing with large claims and catastrophic events, it seems more realistic to consider a 5- or 10years horizon time with a ruin probability respectively around $2.5 \%$ or $5 \%$. We propose for this application to deal with a 5 -years period with a ruin probability around $2.5 \%$. We also suppose that the environment process begins in the soft phase and does not go back to it. This last hypothesis of not going back to the soft phase could be seen as restrictive but is in fact quite realistic. Indeed, our studying time horizon is smaller than the average underwriting cycle length of 6 years as explained in Weiss (2007), Cummins and Outreville (1987) or Venezian (1985). The phases differ here only by their premium income rates, a low one for the soft phase and a high one for the hard phase.

\subsection{Data simulation}

We first simulate 5000 realizations from a Generalized Pareto Distribution (GPD) with cumulative distribution function (c.d.f.), for $x>0$,

$$
F_{G P D}(x)=1-\left(1+\frac{\xi x}{\sigma}\right)^{-\frac{1}{\xi}},
$$

where $\sigma>0$ is the scale parameter and $\xi>0$ the shape parameter. This last parameter is also referred as the extreme value parameter in extreme value theory where $\alpha=1 / \xi$ is called the index of the regular variation of the distribution. This distribution is heavy-tailed and permits to modelize large claims and catastrophic events as fire losses, wind storm, earthquake, etc. The smaller the index $\alpha$ and, consequently, the greater the shape parameter $\xi$, the heavier the right queue of the distribution. For this illustration, we set $\alpha=2.5(\xi=1 / 2.5)$ and $\sigma=1$. A histogram for the simulated data is given in Figure 2. The empirical mean and variance are here respectively 1.63130 
and 11.06983 .

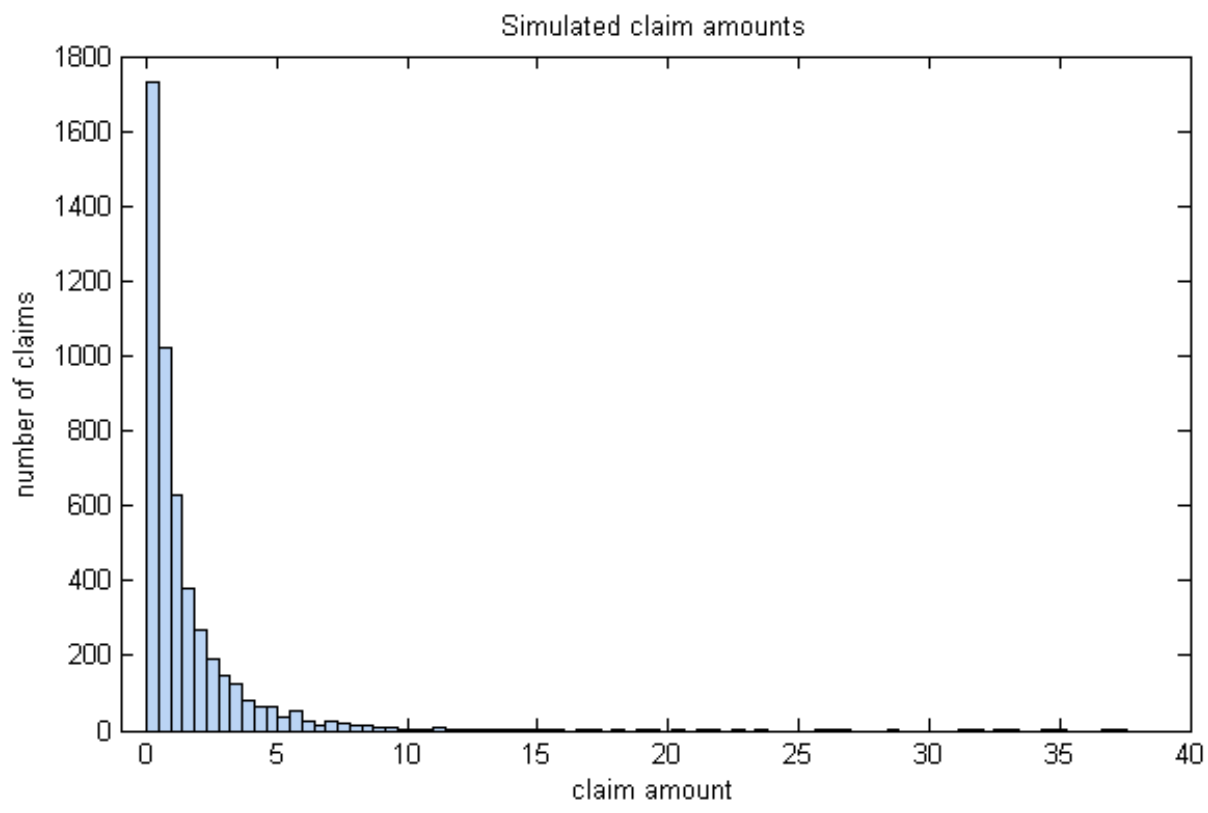

Figure 2: Simulation of claim amounts from a GPD

\subsection{Phase-type fitting}

Our model requires claim amounts to be phase-type distributed. There are different propositions that have be done in the literature to fit phase-type distributions to heavy-tailed distribution or data sets. One could object that this does not allow for heavy tails. However, as we are interested in ruin probabilities of order $2.5 \%$ or $5 \%$ (for time horizon 5 years or 10 years), we are not necessarily in the domain of asymptotic approximation and it is possible to have an appropriate fit of the claim size distribution up to the required level in our case. If we wanted to study ruin probabilities of magnitude $10^{-4}$, this method would of course be inappropriate, and one has to be very cautious when using light-tailed models in risk theory. For example, Feldmann and Whitt (1998) proposed a heuristic-based approach to fit heavy-tailed distribution functions. But this necessitates to first fit an heavy-tailed distribution to the data set which introduces additional errors. The popular expectation-maximization (EM) algorithm permits to fit directly data sets. Several variants of fitting methods using the EM algorithm to obtain a general or a particular phase-type distribution have been explored as in Asmussen and Bladt (1996), Khayari et al. (2003) or Riska et al. (2004). For this application, we use the EM algorithm developed in Lee and Lin (2009) to fit mixtures of Erlang distributions with common scale parameter to heavy-tailed data sets. As written in this reference, this class of mixtures of Erlang distributions with common scale parameter is dense in the space of positive continuous distributions and then can approximate any positive absolutely continuous distribution to any accuracy. Moreover, this class is closed in mixing, convolution, compounding and aggregation of data which offers a good tractability for actuarial purposes. Wang et al. (2006) built an EM fitting algorithm for mixtures of Erlang distributions with common shape parameter and Thummler et al. (2006) with no required common parameter. But these two classes does not 
fulfill the closure properties mentioned above.

The density of a mixture of $k$ Erlang distributions with common scale parameter $\theta>0$ is

$$
f(x)=\sum_{j=1}^{k} \alpha_{j} \frac{x^{r_{j}-1 e^{-x / \theta}}}{\theta^{r_{j}}\left(r_{j}-1\right) !},
$$

where $r_{j}>0$ and $\alpha_{j}>0$ are respectively the shape parameter and the weight of the $j$ th Erlang distribution. The distribution weights must observe the following constraint $\sum_{j=1}^{k} \alpha_{j}=1$.

Applying the Lee \& Lin algorithm to our simulated data set, it results that a density using a mixture of 14 Erlangs fits the empirical density very satisfactorily. The common scale parameter is evaluated at $\theta=0.81585$ and the shape parameters and weights for every Erlang distributions of the mixture are presented in Table 2. This table also displays the expected value associated for each Erlang r.v. $Y_{j}$ of the distribution mixture.

\begin{tabular}{|c|c|c|c||c|c|c|c|}
\hline$j$ & $r_{j}$ & $\alpha_{j}$ & $E\left[Y_{j}\right]$ & $j$ & $r_{j}$ & $\alpha_{j}$ & $E\left[Y_{j}\right]$ \\
\hline 1 & 75 & 0.00063 & 61.18865 & 8 & 22 & 0.00122 & 17.94867 \\
\hline 2 & 59 & 0.00021 & 48.13507 & 9 & 16 & 0.00469 & 13.05358 \\
\hline 3 & 48 & 0.00012 & 47.31922 & 10 & 15 & 0.00283 & 12.23773 \\
\hline 4 & 40 & 0.00199 & 32.63395 & 11 & 14 & 0.00166 & 11.42188 \\
\hline 5 & 39 & 0.00024 & 31.81810 & 12 & 8 & 0.03157 & 6.52679 \\
\hline 6 & 25 & 0.00078 & 20.39622 & 13 & 4 & 0.14131 & 3.26339 \\
\hline 7 & 24 & 0.00122 & 19.58037 & 14 & 1 & 0.81155 & 0.81585 \\
\hline
\end{tabular}

Table 2: Parameters of the fitted mixture of Erlang distributions.

To attest to the goodness of fit, a graphical comparison between a relative histogram for the simulated data and the fitted distribution can be seen in Figure 3, a pp-plot and a qq-plot are drawn in Figure 4. All these graphical tools already show that the fit is very satisfying even in the right queue. A Kolmogorov-Smirnov test is used to confirm the high quality of the fit. Indeed, with a test statistic of 0.00578 and a cutoff value of 0.01917 , the p-value is evaluated at 0.99612 and the hypothesis that the data sample comes from the fitted distribution is not rejected. Again, this method could be dangerous if it were applied to the study of more extreme events.

\subsection{Ruin probability approximation}

We now use our model to evaluate the ruin probability for a horizon $T=5$ years where the claim amounts are distributed according to the fitted mixture of Erlang distributions. Let the inter-claim time random variable $W$ be exponentially distributed with parameter $\lambda=2$. So that, the average time between two claim arrivals is 0.5 year and two claims per year are expected. As for the claim amounts, we suppose that the inter-claim time r.v. are identically distributed in the two phases of 


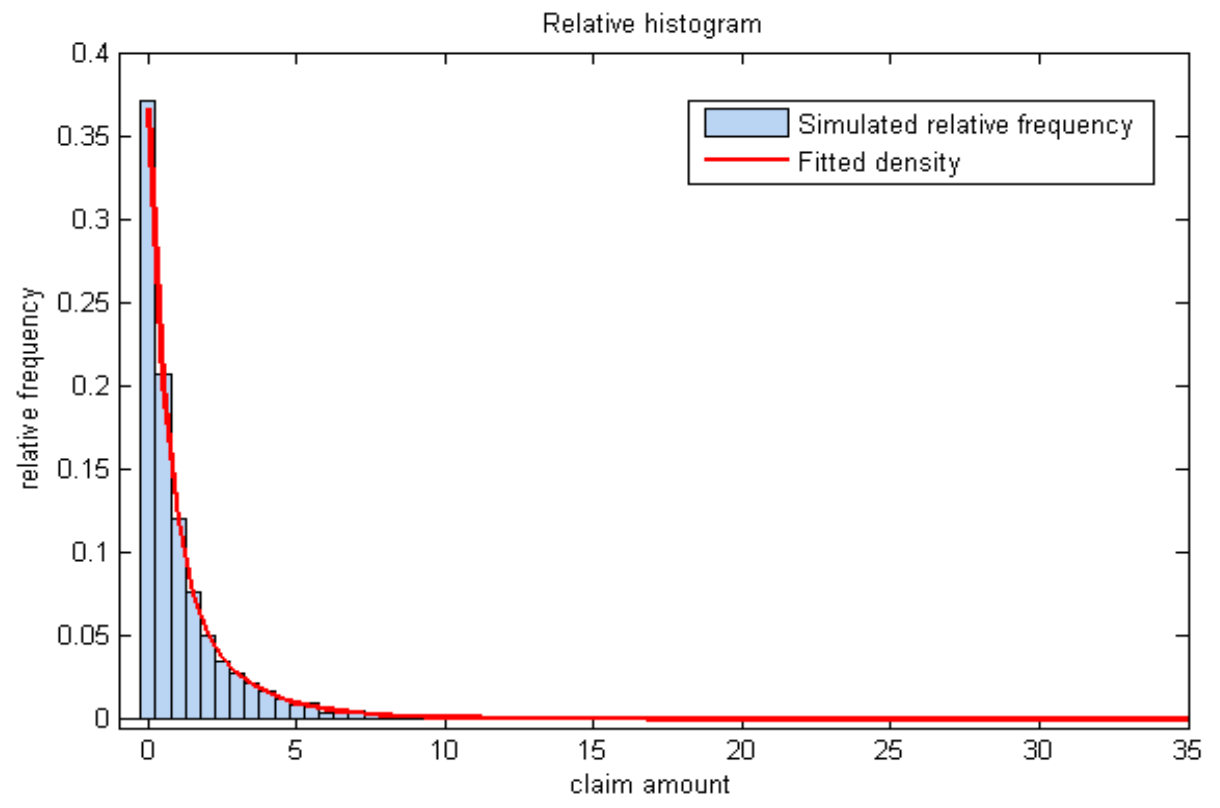

Figure 3: Comparison between a relative histogram for the simulated data and the fitted density
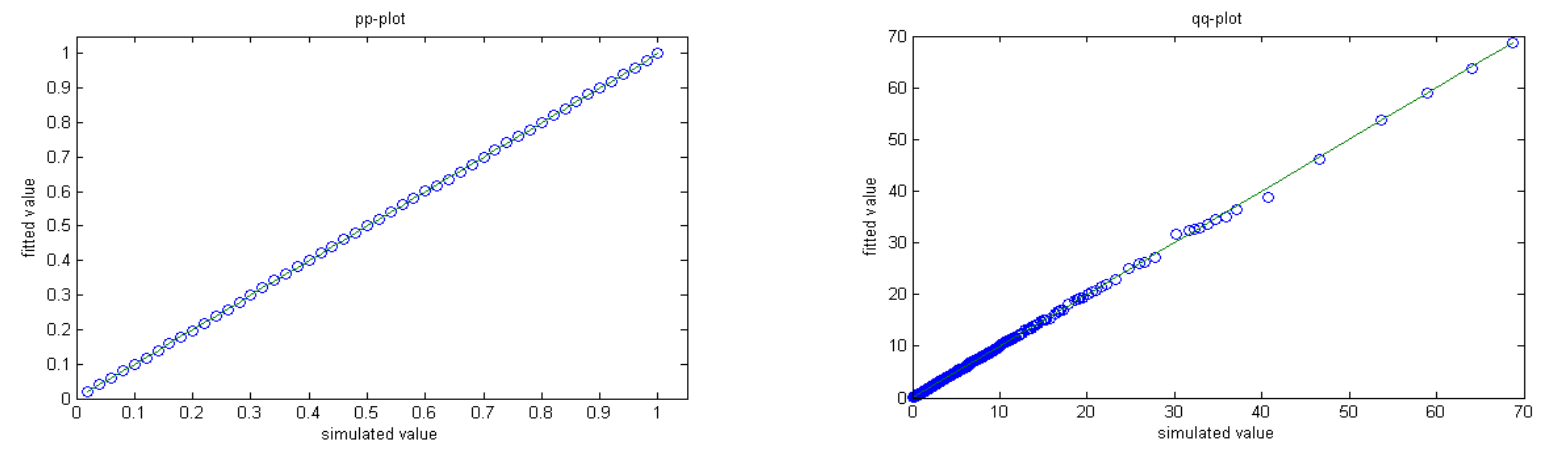

Figure 4: pp-plot and qq-plot for the simulated data versus the fitted distribution 
the cycle. Only the premium income rates differs between the two phases. Indeed, we define the premium rate $c_{i}$, with $i=1$ for the soft phase and $i=2$ for the hard phase, such as

$$
c_{i}=\left(1+\rho_{i}\right) \times E\left[\widehat{S}_{1}\right]=\left(1+\rho_{i}\right) \times E[W] E\left[\widehat{X}_{1}\right]
$$

where $\rho_{i}$ is the security loading corresponding to the phase $i, \widehat{S}_{t}$ is the surplus process where the claim amount random variables $\widehat{X_{k}}$ are i.i.d. with the empirical simulated distribution. Froot (2001) shows that in the market of large claims and catastrophic events the reinsurance premiums can be more than seven times the expected loss during a period. Following this idea, we set $\rho_{1}=30 \%$ and $\rho_{2}=300 \%$. We fix the initial surplus at $u=30$ and the claim amount threshold $x_{0}>0$ that provokes the passage from the soft phase to the hard phase at $x_{0}=25$. This last value represents the $99.663 \%$ quantile of the empirical cumulative distribution. Over the 5 years horizon with 2 claims per year, this does not seem to have an impact the ruin probability. In Table 3 is displayed the probability $p_{1 j}$ of causing a change from the soft cycle to the hard cycle for the $j t h$ Erlang distribution of the mixture, $j=1, \ldots, 14$, as they are defined in Equation (5).

\begin{tabular}{|c|c||c|c|}
\hline$j$ & $p_{1 j}$ & $j$ & $p_{1 j}$ \\
\hline 1 & $\approx 1$ & 8 & 0.04326 \\
\hline 2 & $\approx 1$ & 9 & 0.00138 \\
\hline 3 & 0.99999 & 10 & 0.00064 \\
\hline 4 & 0.94060 & 11 & 0.00028 \\
\hline 5 & 0.91826 & 12 & $\approx 0$ \\
\hline 6 & 0.13159 & 13 & $\approx 0$ \\
\hline 7 & 0.09433 & 14 & $\approx 0$ \\
\hline
\end{tabular}

Table 3: Phase change probabilities of the Erlang distributions of the fitted mixture

We can now construct the required matrices $\Lambda, R_{--}$and $M^{*}$ and use Equations (6), (8) and Theorem 1 to approximate the finite-time ruin probability $\psi(u, T)$. For comparison issues, MonteCarlo (MC) simulations for $\psi(u, T)$ are performed using both empirical and fitted distributions for the claim amounts. The results for the three approximations are given in Table 4 where we used for our method a number of Erlang stages $L=50$.

\begin{tabular}{|c|c|}
\hline Method & Approximation for $\psi(u, T)$ \\
\hline Model 3 & $2.37199 \%$ \\
\hline MC sim. with fitted distr. & $2.35448 \%$ \\
\hline MC sim. with empirical distr. & $2.34998 \%$ \\
\hline
\end{tabular}

Table 4: Approximated finite time ruin probabilities

We see on this Table that our approximation is quite accurate with a relative error of $0.73 \%$ from the Monte-Carlo simulation with the fitted distribution and $0.92 \%$ from the Monte-Carlo simulation with the empirical distribution. The error inserted from the distribution fitting is $0.19 \%$. The computational time required for our model is here around 100 times less than the one needed for the two simulation methods.

The previous example is the reference parametrization that is used in the next subsection. 
But, we also give now some results in Table 5 when modifying one parameter. We see that our approximations are sill very close to the Monte-Carlo simulations obtained with the empirical claim size distributions.

\begin{tabular}{|c|c|c|c|}
\hline Parameter modified & MC simulation & Model 3 & Relative error \\
\hline$x_{0}=30$ & $2.42243 \%$ & $2.42480 \%$ & $0.098 \%$ \\
\hline$\rho_{2}=200 \%$ & $2.38310 \%$ & $2.40007 \%$ & $0.707 \%$ \\
\hline
\end{tabular}

Table 5: Approximated finite time ruin probabilities with a modified parameter

\subsection{Comparison between model 3 and independent changes of cycle phase}

We illustrate now the impact of dependence between phase changes and claim amounts. We compare our model to a so-called independent model in which the change of phase occurs independently from claim amounts. For this independent model, we construct an intensity matrix $\widehat{\Theta}$ for the environment Markov process $J_{t}$ in which the change from the soft phase to the hard phase can appear at any state of the Erlang horizon time $\mathcal{H}$ with probability $p_{0} \in[0,1]$. This is done by modifying the block matrix $\widehat{\Theta}_{--}$such that at any state $i, i=1, \ldots, L-1$ of the Erlang time in the soft cycle phase, the process can go to state $i+1$ of the Erlang time in the hard cycle phase with probability $p_{0}$. Therefore, the change of phase is independent from the claim amount.

For exponentially distributed inter-claim times with parameter $\lambda$ and for an Erlang time horizon $\mathcal{H} \sim \operatorname{Erlang}(2, a)$, the block $\widehat{\Theta}_{--}$becomes

$$
\widehat{\Theta}_{--}=\left(\begin{array}{cc|cc}
-\lambda-a & 0 & a\left(1-p_{0}\right) & a p_{0} \\
0 & -\lambda-a & 0 & a \\
\hline 0 & 0 & -\lambda-a & 0 \\
0 & 0 & 0 & -\lambda-a
\end{array}\right)
$$

and can also be written as

$$
\widehat{\Theta}_{--}=\left(\begin{array}{cc}
\Lambda_{--}-a I & M^{*} \\
0 & \Lambda_{--}-a I
\end{array}\right)
$$

where, as in Subsection 4.2, $M^{*}$ contains the direct transitions between the phases. This can, of course, also be extended to a number of Erlang stages $L \geqslant 2$.

In order to compare this independent model to our dependent model, we give to these two models the same probability to change at least one time from the soft phase to the hard phase. For the independent model, this probability is $P_{0}=1-\left(1-p_{0}\right)^{L}$. In our dependent model, the probability to go to the hard phase after each claim is

$$
\sum_{j=1}^{n} \alpha_{j} p_{1 j}
$$


Then the probability to have no change from the soft phase to the hard phase during $[0, T]$ is

$$
E\left[\left(1-\sum_{j=1}^{n} \alpha_{j} p_{1 j}\right)^{N}\right]
$$

where $N \sim \operatorname{Poisson}(T \lambda)$. Finally, the probability to change from the soft phase to the hard phase at least one time during $[0, T]$ is

$$
P=1-E\left[\left(1-\sum_{i=1}^{n} \alpha_{i} p_{i}\right)^{N}\right]=P_{0}
$$

and we obtain

$$
p_{0}=1-E\left[\left(1-\sum_{i=1}^{n} \alpha_{i} p_{i}\right)^{N}\right]^{1 / L}
$$

We also construct an intermediate model such that the probability to change from the soft phase to the hard phase at least one time is still the same where the cycle can change either independently from claim amounts or because of a large claim. This intermediate model permits to observe the slide between the dependent and independent models and to see the impact of the claim amount dependence on the finite-time ruin probability. The probability to change from the soft phase to the hard phase at least one time during $[0, T]$ for this intermediate model is

$$
P(\theta)=1-\left((1-p(\theta))^{L}\right)\left(E\left[\left(1-\sum_{i=1}^{n} \theta \alpha_{i} p_{i}\right)^{N}\right]\right)=P_{0}
$$

where $\theta \in[0,1]$ and $p(\theta)$ represents the part of the phase change probability that is independent from the claim amounts. Then we obtain

$$
p(\theta)=1-\left(1-p_{0}\right) \times\left(E\left[\left(1-\sum_{i=1}^{n} \theta \alpha_{i} p_{i}\right)^{N}\right]\right)^{-1 / L} .
$$

The bounds $p(0)=p_{0}$ and $p(1)=0$ correspond respectively to the independent model and the dependent model.

For our application, as we use the Richardson extrapolation recalled in Equation (9) for a number of Erlang stages $L=50$, two probabilities $p_{0}$ are evaluated. For $L=50$, we obtain $p_{0}=6.63365 \times 10^{-4}$ and for $L=51, p_{0}=6.50362 \times 10^{-4}$. Thus, the ruin probability is approximated at $2.50960 \%$ with the independent model versus an evaluation at $2.37199 \%$ for our dependent model. We recall that the MC simulation with the empirical distribution provides a finite-time ruin probability at $2.34998 \%$. The error from the independent model on our dependent model can be evaluated at $5.48 \%$ and the one on the MC-simulation at $6.36 \%$. We can imagine that this difference would be quite larger when looking at a greater time horizon but also when including more phases in a cycle. Figure 5 are drawn the slide of the ruin probability with the intermediate model and the slide of the underlying error made comparing to our dependent model. 

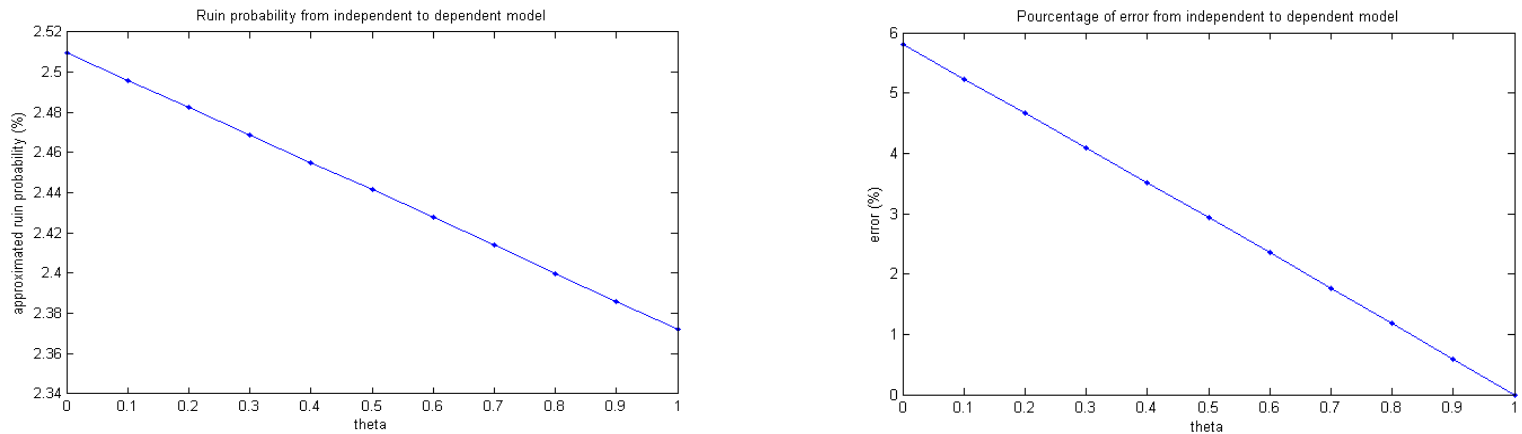

Figure 5: Comparaison between the model 3 and the independent model

\section{Conclusion}

This paper provides an extension for the Erlangization method taking into account the reinsurance cycles in order to evaluate the finite-time ruin probability in the Sparre-Andersen model with phasetype distributed claim amounts and inter-claim times. We assume the presence of two phases in a reinsurance cycle, a soft phase and a hard phase. We first propose a naive model with deterministic changes between the two phases. Then, a more realistic model is developed where the change from the soft phase to the hard phase depends on the severity of the claim amounts. We illustrate our model by an application and compare our results to Monte-Carlo simulations and to the case where the claim amounts have no impact on the phase changes. We show that this feedback control may change the ruin probability by $6 \%$ in our particular numerical example.

\section{Acknowledgements}

This work has been partly funded by the research chair Management de la Modélisation sponsored by BNP Paribas Assurance, the research chair Actuariat Durable sponsored by Milliman, and the French Research National Agency (ANR) under the reference ANR-08-BLAN-0314-01. 


\section{References}

Albrecher, H., Asmussen, S., and Kortschak, D. (2006). Tail asymptotics for the sum of two heavy-tailed dependent risks. Extremes, 9(2):107-130.

Albrecher, H. and Boxma, O. J. (2004). A ruin model with dependence between claim sizes and claim intervals. Insurance Math. Econom., 35(2):245-254.

Albrecher, H. and Teugels, J. L. (2006). Exponential behavior in the presence of dependence in risk theory. J. Appl. Probab., 43(1):257-273.

Asmussen, S. (1995). Stationary distributions for fluid flow models with or without Brownian noise. Comm. Statist. Stochastic Models, 11(1):21-49.

Asmussen, S., Avram, F., and Usabel, M. (2002). Erlangian approximations for finite-horizon ruin probabilities. Astin Bull., 32(2):267-281.

Asmussen, S. and Bladt, M. (1996). Phase-type distributions and risk processes with statedependent premiums. Scand. Actuar. J., (1):19-36.

Avram, F. and Usabel, M. (2003). Finite time ruin probabilities with one Laplace inversion. Insurance Math. Econom., 32(3):371-377.

Badescu, A. and Landriault, D. (2009). Applications of fluid flow matrix analytic methods in ruin theory, a review. Revista de la Real Academia de Ciencias Exactas, Físicas y Naturales. Serie A: Matemáticas (RACSAM), 103(2):353-372.

Biard, R., Lefèvre, C., and Loisel, S. (2008). Impact of correlation crises in risk theory: asymptotics of finite-time ruin probabilities for heavy-tailed claim amounts when some independence and stationarity assumptions are relaxed. Insurance Math. Econom., 43(3):412-421.

Biard, R., Lefèvre, C., Loisel, S., and Nagaraja, H. (2011). Asymptotic finite-time ruin probabilities for a class of path-dependent claim amounts using Poisson spacings. Applied Stochastic Models in Business and Industry, to appear.

Boudreault, M., Cossette, H., Landriault, D., and Marceau, E. (2006). On a risk model with dependence between interclaim arrivals and claim sizes. Scand. Actuar. J., (5):265-285.

Cummins, J. and Outreville, J. (1987). An international analysis of underwriting cycles in propertyliability insurance. Journal of Risk and Insurance, pages 246-262.

Cummins, J. D., Dionne, G., Gagné, R., and Nouira, A. (2008). The costs and benefits of reinsurance. Cahiers de recherche 08-04, HEC Montréal, Institut d'économie appliquée.

De Vylder, F. E. and Goovaerts, M. J. (1999). Explicit finite-time and infinite-time ruin probabilities in the continuous case. Insurance Math. Econom., 24(3):155-172.

Feldmann, A. and Whitt, W. (1998). Fitting mixtures of exponentials to long-tail distributions to analyze network performance models. Performance Evaluation, 31(3-4):245-279.

Froot, K. (2001). The market for catastrophe risk: a clinical examination. Journal of Financial Economics, 60(2-3):529-571. 
Graham, A. (1981). Kronecker products and matrix calculus: with applications. Ellis Horwood Ltd., Chichester. Ellis Horwood Series in Mathematics and its Applications.

Hardy, M. R. (2001). A regime-switching model of long-term stock returns. N. Am. Actuar. J., $5(2): 41-53$.

Ignatov, Z. G. and Kaishev, V. K. (2004). A finite-time ruin probability formula for continuous claim severities. J. Appl. Probab., 41(2):570-578.

Khayari, R. E. A., Sadre, R., and Haverkort, B. R. (2003). Fitting world-wide web request traces with the EM-algorithm. Performance Evaluation, 52(2-3):175 - 191. Internet Performance and Control of Network Systems.

Kortschak, D. and Albrecher, H. (2009). Asymptotic results for the sum of dependent nonidentically distributed random variables. Methodol. Comput. Appl. Probab., 11(3):279-306.

Lee, S. and Lin, S. (2009). Modelling insurance losses and calculating risk measures via a mixture of Erlangs. Working paper, University of Toronto.

Lefèvre, C. and Loisel, S. (2009). Finite-time ruin probabilities for discrete, possibly dependent, claim severities. Methodol. Comput. Appl. Probab., 11(3):425-441.

Picard, P. and Lefèvre, C. (1997). The probability of ruin in finite time with discrete claim size distribution. Scand. Actuar. J., (1):58-69.

Ramaswami, V., Woolford, D. G., and Stanford, D. A. (2008). The Erlangization method for Markovian fluid flows. Ann. Oper. Res., 160:215-225.

Riska, A., Diev, V., and Smirni, E. (2004). An EM-based technique for approximating long-tailed data sets with PH distributions. Performance Evaluation, 55(1-2):147-164.

Rullière, D. and Loisel, S. (2004). Another look at the Picard-Lefèvre formula for finite-time ruin probabilities. Insurance Math. Econom., 35(2):187-203.

Sidje, R. B. (1998). ExPOKIT. A software package for computing matrix exponentials. ACM Trans. Math. Softw., 24(1):130-156.

Stanford, D. A., Avram, F., Badescu, A. L., Breuer, L., da Silva Soares, A., and Latouche, G. (2005a). Phase-type approximations to finite-time ruin probabilities in the Sparre-Andersen and stationary renewal risk models. Astin Bull., 35(1):131-144.

Stanford, D. A., Latouche, G., Woolford, D. G., Boychuk, D., and Hunchak, A. (2005b). Erlangized fluid queues with application to uncontrolled fire perimeter. Stoch. Models, 21(2-3):631-642.

Thummler, A., Buchholz, P., and Telek, M. (2006). A novel approach for phase-type fitting with the EM algorithm. IEEE Transactions on Dependable and Secure Computing, 3(3):245.

Venezian, E. (1985). Ratemaking methods and profit cycles in property and liability insurance. Journal of Risk and Insurance, pages 477-500.

Wang, J., Zhou, H., Zhou, M., and Li, L. (2006). A general model for long-tailed network traffic approximation. J. Supercomput., 38(2):155-172. 
Weiss, M. A. (2007). Underwriting cycles: a synthesis and further directions. Astin Bull., $(30(1)): 31-45$.

Wüthrich, M. V. (2003). Asymptotic value-at-risk estimates for sums of dependent random variables. Astin Bull., 33(1):75-92.

Yu, K., Stanford, D. A., and Ren, J. (2007). Erlangian approximation to finite time ruin probabilities in perturbed risk models. In Bini, D., Meini, B., Ramaswami, V., Remiche, M.-A., and Taylor, P. G., editors, Numerical Methods for Structured Markov Chains, volume 07461 of Dagstuhl Seminar Proceedings. Internationales Begegnungs- und Forschungszentrum fuer Informatik (IBFI), Schloss Dagstuhl, Germany. 


\section{APPENDIX}

\section{A Matrix inversion}

The Erlangization method used to evaluate the ruin probability or equivalently the probability for the fluid model to reach 0 before the horizon $\mathcal{H}$ requires the inversion of several matrices. As seen in Theorem 1, the matrix of interest $\eta$ can be approximated by the iteration scheme $\eta=\lim _{n \rightarrow \infty} \eta^{(n)}$ where $\eta^{(0)}=0$ and

$$
\begin{aligned}
\eta^{(n+1)} & =\left(\left(\delta I+\Theta^{--}\right) \eta^{(n)}+\Theta^{-+}\right)\left(\delta I-\left(\Theta^{++}+\Theta^{+-} \eta^{(n)}\right)\right)^{-1} \\
& =A^{(n)} \times\left(B^{(n)}\right)^{-1}
\end{aligned}
$$

Thus, to determine $\eta^{(n+1)}$, we need to inverse the matrice $B^{(n)}$. The Erlangization method, according to the specific structure of the intensity matrix of the Erlang horizon $\mathcal{H}$, provides that the block matrices $\Theta^{--}, \Theta^{-+}, \Theta^{+-}$and $\Theta^{++}$have all an upper triangular block Toeplitz structure and that the matrix $B^{(n)}$ takes this form

$$
B^{(n)}=\left(\begin{array}{ccccc}
B_{1}^{(n)} & B_{2}^{(n)} & B_{3}^{(n)} & \ldots & B_{L}^{(n)} \\
0 & B_{1}^{(n)} & B_{2}^{(n)} & \ldots & B_{L-1}^{(n)} \\
0 & 0 & B_{1}^{(n)} & \ldots & B_{L-2}^{(n)} \\
\vdots & \vdots & \vdots & \ddots & \vdots \\
0 & 0 & 0 & \ldots & B_{1}^{(n)}
\end{array}\right) .
$$

Indeed, this structure is stable for addition, multiplication and inversion and $\left(B^{(n)}\right)^{-1}, A^{(n)}$ and $\eta^{(n)}$ also have the form.

Let $M$ be a $m n \times m n$ matrix such as

$$
M=\left(\begin{array}{ll}
M_{1} & M_{2} \\
M_{3} & M_{4}
\end{array}\right)
$$

where the $m \times m$ block matrix $M_{1}$ and the $n \times n$ block matrix $M_{4}$ are invertible. Then, we have

$$
M^{-1}=\left(\begin{array}{cc}
\left(M_{1}-M_{2} M_{4}^{-1} M_{3}\right)^{-1} & -M_{1}^{-1} M_{2}\left(M_{4}-M_{3} M_{1}^{-1} M_{2}\right)^{-1} \\
-M_{4}^{-1} M_{3}\left(M_{1}-M_{2} M_{4}^{-1} M_{3}\right)^{-1} & \left(M_{4}-M_{3} M_{1}^{-1} M_{2}\right)^{-1}
\end{array}\right) .
$$

To inverse the matrix $B^{(n)}$ described before, we can split it into four blocks as follows:

$$
B^{(n)}=\left(\begin{array}{cc}
M_{1} & M_{2} \\
M_{3} & M_{4}
\end{array}\right) \text {, }
$$


where

$$
\begin{gathered}
M_{1}=\left(\begin{array}{cccc}
B_{1}^{(n)} & B_{2}^{(n)} & \ldots & B_{L-1}^{(n)} \\
0 & B_{1}^{(n)} & \ldots & B_{L-2}^{(n)} \\
\vdots & \vdots & \ddots & \vdots \\
0 & 0 & \ldots & B_{1}^{(n)}
\end{array}\right), \quad M_{2}=\left(\begin{array}{c}
B_{L}^{(n)} \\
B_{L-1}^{(n)} \\
\vdots \\
B_{2}^{(n)}
\end{array}\right), \\
M_{3}=\left(\begin{array}{llll}
0 & 0 & \ldots & 0
\end{array}\right) \text { and } M_{4}=\left(B_{1}^{(n)}\right)
\end{gathered}
$$

Thus we have

$$
\left(B^{(n)}\right)^{-1}=\left(\begin{array}{cc}
M_{1}^{-1} & -M_{1}^{-1} M_{2} M_{4}^{-1} \\
0 & M_{4}^{-1}
\end{array}\right) .
$$

The remaining difficulty stays in the inversion of the block matrix $M_{1}$. To this aim, we proceed algorithmically using the same procedure as for $B^{(n)}$.

\section{B Initialization for the deterministic phase change instants model}

For the computation of the approximation of the ruin probability in the case of deterministic phase change instants, we have some technical but not stopping constrains on the numbers of states $L_{1}$ for $\mathcal{H}_{1}$ and $L_{2}$ for $\mathcal{H}_{2}$. Indeed, we have

$$
\begin{aligned}
& \mathcal{H}_{1} \sim \operatorname{Erlang}\left(L_{1}, a\right), \\
& \mathcal{H}_{2} \sim \operatorname{Erlang}\left(L_{2}, a\right) .
\end{aligned}
$$

The final horizon time $T$ can be decomposed as $T=T_{1}+T_{2}$ such as

$$
\begin{aligned}
& T_{1}=E\left[\mathcal{H}_{1}\right]=\frac{L_{1}}{a}, \\
& T_{2}=E\left[\mathcal{H}_{2}\right]=\frac{L_{2}}{a} .
\end{aligned}
$$

Both $T_{1}$ and $T_{2}$ are fixed and then we have to find appropriate values for $a \in \mathbb{R}_{+}^{*}, L_{1} \in \mathbb{N}$ et $L_{2} \in \mathbb{N}$ such that

$$
\begin{aligned}
L_{1} & =a T_{1}, \\
L_{2} & =a T_{2}, \\
L=L_{1}+L_{2} & =a\left(T_{1}+T_{2}\right)=a T .
\end{aligned}
$$

These equalities can be fulfilled by choosing reasonable values for $L, L_{1}$ and $L_{2}$ knowing that improving this values leads to better approximations. 